\title{
COVID-19 and Pregnancy: Risk, Symptoms, Diagnosis, and Treatment
}

\author{
Vera Hapshy ${ }^{1} \cdot$ Daniel Aziz $^{2} \cdot$ Payal Kahar $^{3} \cdot$ Deepesh Khanna ${ }^{1} \cdot$ Kenneth E. Johnson ${ }^{1} \cdot$ Mayur S. Parmar $^{1}$ (i)
}

Accepted: 13 April 2021 / Published online: 21 April 2021

(C) The Author(s), under exclusive licence to Springer Nature Switzerland AG 2021

\begin{abstract}
Severe acute respiratory syndrome coronavirus-2 (SARS-CoV-2) is a novel virus responsible for causing an infection known as COVID-19. Several pulmonary and systemic manifestations of the illness have been described since the discovery of this virus. However, there have been higher-risk populations in which this infection has not been well studied nor documented. One of these populations includes the pregnant cohort. The purpose of this article is to describe the clinical manifestations of COVID-19 infection in the pregnant population and review the implications and sequelae of the infection throughout pregnancy and outcomes of live births. Also, we summarize the understanding and safety of current treatments and vaccination in pregnancy. This comprehensive review article comprises several case reports, case series, cohort studies, retrospective studies, and randomized clinical trials. Findings regarding maternal morbidity included an increased risk of acquiring severe COVID-19 infection requiring a higher level of inpatient hospital care along with an increased risk of preterm labor and cesarean delivery. Neonatal COVID-19 vertical transmission was shown to have conflicting data as there was a presence of transmission in certain retrospective studies and absence in others. There was also no evidence of teratogenicity from maternal COVID-19 infection. In conclusion, in part due to the unique physiologic state of pregnancy and part due to unknown factors, pregnant patients are at increased risk for negative outcomes of COVID-19 infection and must be classified as a high-risk population.
\end{abstract}

Keywords COVID-19 $\cdot$ SARs-CoV-2 $\cdot$ Pregnancy $\cdot$ Neonatal outcomes $\cdot$ Placenta $\cdot$ Vertical transmission $\cdot$ Treatment $\cdot$ Vaccine

\section{Introduction}

In December 2019, a novel form of the coronavirus was identified in the Wuhan Province of China. The novel coronavirus, renamed SARS-COV-2, causes an infectious disease called COVID-19. Since the identification in December in China, there have been over 136 million cases worldwide and over 31 million cases in the United States as of April 12, 2021 [1]. This condition causes a wide array of symptoms spanning organ systems and mimicking many other disease states. COVID-19 has been proven in many systematic reviews and studies to affect vulnerable populations such as

This article is part of the Topical Collection on Covid-19

Mayur S. Parmar

mparmar@nova.edu

1 Dr. Kiran C. Patel College of Osteopathic Medicine, Nova Southeastern University, Tampa Bay Campus, Clearwater, FL, USA

2 Rutgers New Jersey Medical School, Newark, NJ, USA

3 Department of Health Sciences, Florida Gulf Coast University, Fort Myers, FL, USA immunocompromised hosts and the elderly. Pregnant patients' susceptibility in the outbreaks of infection with the severe acute respiratory syndrome (SARS) discovered in 2004 provides evidence that this population may also be at increased risk from COVID-19 infection [2]. The unique physiologic changes in pregnancy which often mimic infectious, and inflammatory etiologies are particularly concerning due to the vast array of symptoms caused by COVID-19. The public outcry and politicization of this disease have also caused fear for many mothers in the early and late gestation stages regarding their health and their growing fetus' health. Many observational studies have pooled data from case reports and hospital registries to obtain susceptibility and outcomes of pregnant patients infected with COVID-19. The purpose of this review article is to gather the current data regarding the presenting signs and symptoms of COVID-19 in pregnancy, distinguish these symptoms from those that present with normal physiologic changes in pregnancy, and establish safe and effective ways to diagnose COVID-19. Alongside this, in this article, we identify possible obstetric complications of COVID-19 in the mother and the neonate and discuss the management options and potential obstetric complexities currently in place. 


\section{Signs and Symptoms}

During much of the gestational period, the pregnant woman undergoes many physiologic changes that mimic multiple pathologies and increase the susceptibility to acquiring infectious diseases. Changes to multiple organ systems occur during pregnancy, most notably in the cardiovascular and respiratory systems. Pregnant women see a rapid increase in blood plasma volume and increased red blood cell mass, peaking in the third trimester [3]. This expansion in blood plasma volume greater than blood cell mass causes physiological dilutional anemia. Anemia in pregnancy is a leading cause of morbidity in pregnancy, causing symptoms of increased fatigue. The associated increase in heart rate and decreased systemic vascular resistance also increases physiologic morbidity through tachycardia and lower extremity edema. Meanwhile, in the respiratory system, patients increase total minute ventilation through an increase in total tidal volume. The increased ventilation causes respiratory alkalosis throughout pregnancy resulting in physiologic dyspnea of pregnancy. It is reported that around 60 to 70 percent of women experience shortness of breath and dyspnea on exertion, increasing in frequency in the second trimester [4].

These physiologic changes make distinguishing pulmonary pathology difficult during pregnancy. This difficulty is heightened by the fact that COVID-19 infection has a wide array of presenting signs of symptoms. COVID-19 symptoms in pregnancy are similar to infections that occur in the general population with respect to presenting symptoms of cough (52\%) and shortness of breath (30\%). Pregnant women, however, were less likely to report symptoms of headache (41\%), fever (34\%), chills (38\%), and diarrhea (14\%) compared to the general population [5]. The other presenting symptoms, although less common, of COVID-19 infection, include loss of appetite, nausea/vomiting, loss of smell, rhinorrhea, sore throat, and abnormal laboratory values of lymphopenia and transaminitis.

It is crucial in the evaluation of a pregnant patient to educate them regarding symptoms that are not associated with normal pregnancy changes. With increased public awareness of COVID infection, patients may be more vigilant regarding symptoms such as shortness of breath and fatigue. It is essential to inform patients to seek evaluation for more distressing symptoms such as any new-onset fever, cough, or a sudden increase in work of breathing and shortness of breath. Figure 1 shows symptoms unique and overlapping for COVID-19 and pregnancy $[2,4,5]$.

\section{Diagnosis}

Diagnosis of COVID-19 should be suspected in patients with new-onset fever and upper or lower respiratory tract symptoms. Any patient who has these symptoms should undergo

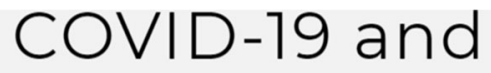

\section{Pregnancy Symptoms}
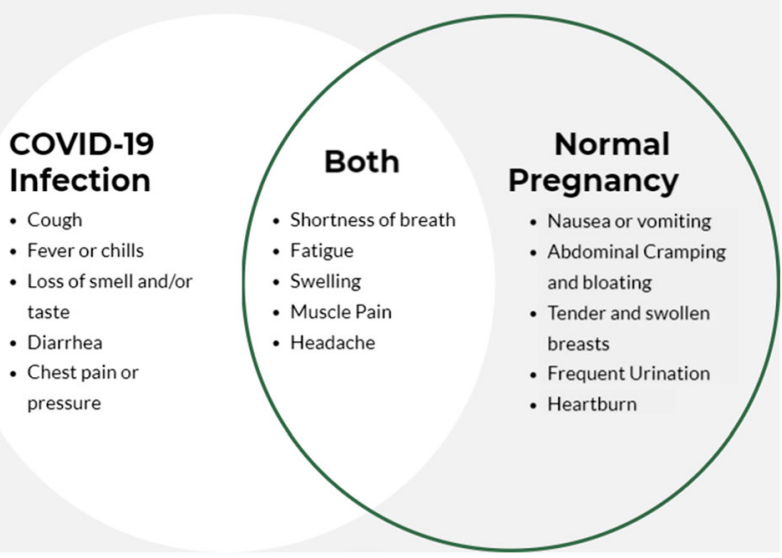

Fig. 1 Symptoms unique and overlapping for COVID-19 and pregnancy $[2,4,5]$

reverse transcriptase-polymerase chain reaction (RT-PCR) testing for SARS-COV-2 RNA through the nasopharyngeal swab. Another testing should be done in conjunction with PCR testing, including respiratory pathogen panel and, depending on seasonality, influenza testing. A positive test confirms the diagnosis of COVID-19 infection. Although there are false-positives rates, the test specificity is almost $100 \%$ due to the RT-PCR sequence. A negative test indicates possible negative infection, but there may be a high ratio falsenegative due to sampling error, type of sample acquired (i.e., nasopharyngeal vs. salivary), and the time between symptom onset and time of testing [6].

Imaging is not required for the diagnosis of infection but is commonly done during the work-up of suspected or positive COVID patients. The predominant imaging done is chest $\mathrm{x}$ ray with follow-up C.T. scan for delineation of severity. Findings on chest $\mathrm{x}$-ray are similar in pregnant and nonpregnant patients, including airspace opacities, multilobar infiltrates, and ground-glass opacities. Consolidation in COVID is seldom lobar and affects the peripheral areas of the lungs [7]. In pregnancy, the C.T. scan is relatively contraindicated due to radiation effects on the developing neonate and should only be used in patients with a higher degree of suspicion or when needed to influence management.

\section{Complications}

It is important to note that when most prospective mothers are infected or afflicted with any illness, they are concerned about their own and their fetuses' health and outcomes. Thus, it is important to identify the effect of COVID-19 infection on maternal, fetal, and neonatal health. Much is presently unknown about COVID-19 in the gestational period due to the 
sudden outbreak of the disease and its lack of research in longer-term observational studies. However, we will describe the current literature regarding maternal and neonatal complications as currently understood.

\section{Maternal Morbidity}

\section{Preterm Labor}

The data on the risk of preterm labor in pregnant patients with COVID-19 as opposed to those without is very mixed. A systematic review of over 350 pregnant patients showed that preterm labor rates in patients $>30$ weeks' gestation were $15 \%$. This rate is increased from the national average of $10 \%$ preterm birth rate in the United States [8]. Another observational study of 55 pregnant patients with infection yielded a rate of $43 \%$ preterm labor in that specific cohort. These patients ranged in age from 23 to 40 years old, and all were infected with COVID-19 in the third trimester [9]. There is thought that the infection and fever may increase the likelihood of preterm labor. There is also a sense of bias in the data, as many providers are more inclined to induce labor in infected patients for fear of unknown or unforeseen complications. There is insufficient data to determine the exact etiology of the cause of preterm labor and whether there is a causal relationship between COVID-19 infection and preterm labor. However, there appears to be an association of third trimester COVID-19 infections and the rate of preterm labor $[8,9]$.

\section{Cesarean Section}

Similar to the rates of preterm labor, the rates of cesarean delivery in patients afflicted with COVID-19 are elevated due to provider preference not to deliver vaginally. An extensive systematic review of over 500 COVID-19 infected pregnant patients reported rates for delivery in 435 patients. The cesarean delivery rate in these patients was at $84.7 \%$; only a reported 8 cases required ICU level of care [5]. This indicates that the cesarean delivery rate was correlated to physician practice and erring on the side of caution rather than required cesarean for maternal and neonatal complications. There were no maternal deaths in this study and only one neonatal death.

Similarly, another cohort study between March and April showed increased cesarean rates from normal in COVID-19infected patients. These patients were stratified from asymptomatic to severe disease based on WHO classification. In the severely infected patients, $52.4 \%$ were delivered via $\mathrm{C}$-section instead of $92 \%$ of the critical patients delivered via $\mathrm{C}$-section. There were decreased rates in asymptomatic and mild cases [10]. This cohort study made an association between the severity of the COVID-19 infected and the mode of delivery with higher rates of cesarean in patients with more severe disease.
Lastly, another systematic review in pregnant patients with greater than $90 \%$ hospitalization rates for COVID-19 pneumonia showed an increased C-section rate of $91 \%$. This study shows the increased rate in severe COVID-19 pneumonia requiring oxygen support and hospitalization and is not representative of most of the population infected with COVID-19 [11].

\section{Post-Partum Hemorrhage}

There is little data regarding the differing rates between postpartum hemorrhage (PPH) in those vaginally delivered with and without COVID-19 serologically identified infection. Due to the hypercoagulable changes associated with pregnancy and COVID-19, there is reason to believe there would be altering PPH rates in patients afflicted. However, early Chinese studies showed there was no increased risk of PPH in patients delivering with COVID-19, although in the study, there was increased usage of oxytocin during and after delivery [12]. In the event of PPH, there has been a suggestion against the use of antifibrinolytic agents, such as tranexamic acid, due to the increased risk of thrombosis involved in severe and critical COVID-19-infected patients. Thus, practitioners may choose against this and other treatments that can cause pulmonary arterial vasoconstriction, such as methylergotamine, due to hypoxia's theoretical risk. There is limited data to support these suggestions or findings [13].

\section{Maternal Mortality}

There was seemingly no increased rate of maternal mortality in multiple systematic reviews and cohort studies in those hospitalized with COVID-19. The mortality rate in pregnant and non-pregnant patients is relatively equal. In two systematic reviews in New York and of selected widespread databases, the mortality rate of over 250 and 350 patients, respectively, was $0.0 \%[5,10]$. In contrast to other coronavirus outbreaks such as SARS and MERS, COVD-19 shows no increased maternal mortality risk. SARS had reported $25 \%$ and MERS a 28\% risk of maternal mortality [11]. There is an increased hospitalization and ICU admissions rate with mechanical ventilation compared to a matched non-pregnant cohort. However, this is not associated with any increased rate of mortality in pregnant patients. There is no known correct number of maternal mortalities currently, but it is believed to be similar to the rate of COVID-19 infection mortality of the general population. In Florida, pregnancy mortality is assessed quarterly by the Pregnancy Associated Mortality Review (PAMR), a statewide maternal mortality review process tasked to better understand why women are dying during pregnancy, childbirth, and the year post-partum. Approximately half the states in the U.S. have a comprehensive maternal mortality review process. 


\section{Neonatal Morbidity}

As important as the effects of COVID-19 infection are on the mother, the infection may also impact the developing fetus. Due to the unknown nature of the coronavirus, infected pregnant patients will often have questions and concerns regarding the effects of coronavirus on their fetus. Most importantly, they will have concern for risk of transmission to the fetus, risk of fetal mutations and malformations, and, ultimately, risk for fetal demise. As per the currently available literature, there seems to be no clear vertical transmission of the virus to the fetus. A small observation study conducted in the U.S. of 43 newborns from infected mothers showed 0 positive results upon testing during the first day of life [14]. In opposition, there is data showing a small positive rate in newborns. In a relatively larger systematic review of 936 positive mothers, there was a $3.2 \%$ COVID-19 PCR positive rate via nasopharyngeal swab (27/936) in neonates. This rate mirrored other studies done in China in 2020 during the outbreak. These rates were for third-trimester maternal COVID-19 infection. These rates were deemed to be similar to pathogens that cause congenital infections. Due to the limited data of first-trimester infections, it is difficult to conclude any early antepartum vertical transmission to the neonate [15]. A lancet observational cohort study done in New York City of over 1400 deliveries, including 116 to COVID-19 positive mothers, showed that no neonates at 24 hours tested positive for coronavirus. Of the 82 neonates who followed up within 5 to 7 days, 68 roomed with their mothers, and 64 were breastfed for the 5 to 7 days of rooming. The repeat COVID-19 PCR was negative in all neonates who were breastfed and who followed up. This suggests that breastfeeding is safe, and there is limited vertical transmission of COVID-19 to neonates. There may be components of inappropriate measures taken in those with positive COVID-19 serologies at 24 hours [16]. This conflicting data makes it difficult for physicians to educate their patients regarding vertical COVID-19 transmission.

However, it is possible to educate patients regarding the potential risks of neonatal COVID-19 infection. The overwhelming majority of data regarding neonatal COVID-19 infections shows mild respiratory distress as the most common complication. A review in the Children's Hospital of Wuhan reported on 33 patients born from COVID-19 positive mothers, three of these patients had COVID-19 infection, and the symptoms included fever with neonatal sepsis and acute hypoxic respiratory failure without cyanosis [17].

\section{Teratogenicity}

Current data suggests that there is no risk of teratogenic effects in developing fetuses due to COVID-19 antepartum infection. Although the data is limited due to limited reviews and small observational studies, preliminary research shows no teratogenicity [18].

\section{Treatment}

Treatment of infectious disease in pregnant patients often presents a unique challenge due to the many known and unknown adverse effects of antimicrobials on the developing fetus. This, coupled with the evolving treatment of COVID-19, makes this challenge even more difficult.

\section{Maternal Stratification and Respiratory Support}

The current understanding and treatment of COVID-19 in non-pregnant patients is often influenced by the severity of the disease, individual comorbidities, degree of immunosuppression of the patient, and various other factors. Many patients are placed on an experimental treatment that shows promising preclinical or early phase clinical trial data. Many of the current pharmaceutical drugs used to treat COVID-19 are not well studied in pregnancy, adding even more reason for hesitancy in prescribing physicians. Thus, severity indexes must be used to stratify COVID-19 positive patients. Many stratification methods used in non-pregnant patients, such as D-dimer levels, Sequential organ failure assessment (SOFA) score, and $\mathrm{O} 2$ saturation, differ in pregnancy due to maternal physiologic changes. Pregnant patients have a hypercoagulable state coinciding with elevated D-dimer levels at baseline. Thus, D-dimer should not be used to stratify the severity of COVID-19 infection in pregnant patients [19]. Oxygen saturation levels also differ in pregnant patients as, at baseline, this population has a higher resting $\mathrm{PaO} 2$ level to increase fetal oxygenation. Thus, there is a higher threshold for dropping oxygen saturation levels. Many physicians use the cutoff of $<92 \%$ or even lower in non-pregnant patients with COVID to begin supplemental oxygen. However, the WHO recommends maintaining an $\mathrm{O} 2$ saturation between $92-95 \%$ and a $\mathrm{PaO} 2>70$ in pregnant patients to decrease the risk of fetal hypoxemia and acidosis [20]. The threshold for mechanical ventilation in pregnancy is also reduced for the reasons mentioned previously. With increasing oxygen supplementation without response and $\mathrm{PaO} 2<70$, evidence suggests early intubation decreases the morbidity and mortality rates of pregnant patients with acute hypoxemic respiratory failure [21].

\section{Thromboembolic Management}

Pregnancy is a known state of hypercoagulability - predisposing patients to venous and arterial thromboembolism. There have been many preliminary reports and observational studies that support an increased risk of thrombosis in patients with COVID-19 [22]. Current evidence supports venous thromboembolism (VTE) prophylaxis with unfractionated or low- 
molecular-weight heparin (LMWH) for all pregnant patients admitted with COVID-19. Five thousand units of unfractionated heparin (UFH) every 12 hours are recommended over LMWH because it is more readily reversible. However, UFH is classified as a pregnancy category $\mathrm{C}$ drug while LMWH falls in category B, which may sway practitioners towards the use of LMWH for anticoagulation in the inpatient setting. LMWH may also be used in cases in which delivery is not expected within days to weeks [23]. There are reports of many hospitals using full-dose anticoagulation in patients with worsening oxygen saturation $<92 \%$ on $>4 \mathrm{~L}$ of supplemental oxygen. However, there is currently minimal data to support therapeutic dose anticoagulation without diagnosed deep vein thrombosis, pulmonary embolism or clinical signs, and symptoms of the clot [24].

\section{Antiviral Therapy}

Remdesivir Remdesivir is the most recent antiviral studied in the treatment of COVID-19. This antiviral is used in patients with severe COVID-19 under study protocol, initially given through compassionate use and more recently approved by the FDA for more widespread administration. This is less so done in pregnant patients as this drug was developed to treat Ebola and Marburg viruses. However, recently accepted manuscript data spanning from March 21 to June 16 described the use of remdesivir in hospitalized pregnant women with confirmed COVID-19 infection and O2 saturation $<94 \%$ who met the criteria to be enrolled in the compassionate use program. Of the 86 patients enrolled in this study, 19 were delivered before their first course of remdesivir and were included in the immediate post-partum group. The remaining patients, whose median gestational age was 28 weeks, were started on remdesivir with follow-up after 28 days. After the 28-day follow-up, the oxygen requirement in $96 \%$ of the pregnant patients decreased.

Furthermore, $93 \%$ of those requiring mechanical ventilation were extubated, 93\% recovered, and 90\% were discharged. Adverse events were experienced in 29\% (22/ 67 ) of the cohort. These adverse events were described as anemia, constipation, dysphagia, worsening hypoxia, and deep vein thrombosis, among others. Other side effects shown included increasing liver function tests and serum creatinine levels with seven pregnant women discontinuing the study drug due to adverse events [25]. Currently, remdesivir is classified as a pregnancy category B2 pharmaceutical drug - epitomizing the limited data of remdesivir in the pregnant cohort. There is little data regarding the fetal effects of remdesivir from its current usage in the treatment of COVID-19 infections or during prior clinical trials to treat Ebola. With consideration of the low molecular weight and avid protein binding, it is hypothesized that remdesivir may cross the placenta, although more clinical studies are needed to determine any adverse neonatal effects [26].

\section{Corticosteroids}

Corticosteroids, such as dexamethasone, are currently indicated in the treatment of severely ill non-pregnant patients who require oxygen or ventilatory support. Corticosteroids are often indicated in pregnant patients who are in or at high risk for preterm labor to promote fetal lung maturity. More recently, there has been data supporting the use of dexamethasone in patients with severe COVID-19 infection. This controlled trial demonstrated that in patients either requiring oxygen support or on mechanical ventilation, there was a decrease in 28-day mortality rates [27]. Dexamethasone or betamethasone is the preferred agents used to induce fetal pulmonary maturation in patients between 24 and 34 weeks of gestation at increased risk for preterm labor. Other corticosteroids that have been used include methylprednisolone or hydrocortisone, as these steroids limit fetal steroid exposure [28]. This theoretical usage has less published data for reducing maternal mortality. All corticosteroids, including dexamethasone, belong to the pregnancy category $\mathrm{C}$ due to the fact that these pharmaceutical drugs cross the placenta and are only partially metabolized by placental enzymes into inactive metabolites [29, 30]. These molecular properties, coupled with the potential teratogenicity of dexamethasone, have given physicians much trepidation. Potential side effects of dexamethasone on the developing fetus include theoretical distortions of osteogenesis in the fetus and fetal malformations such as oral clefts and intrauterine fetal growth restriction [31]. Despite these possible adverse outcomes, the American College of Obstetricians and Gynecologists (ACOG) recommends that treatment with dexamethasone should not be withheld in pregnant patients when indicated [28]. Thus, it is recommended to use dexamethasone in pregnant patients in the ICU or mechanically ventilated patients due to the benefits shown in the treatment of COVID-19 and for the maturing neonate.

\section{Vaccination}

With the recent release of the mRNA vaccines, PfizerBioNtech (BNT162b2) and Moderna (mRNA-1273), there is concern from pregnant patients regarding the vaccine's detrimental effects in utero. However, mRNA vaccines do not contain the live virus that causes COVID-19; therefore, COVID-19 cannot be contracted from the vaccine. Additionally, mRNA vaccines do not interact with one's DNA because the mRNA does not enter the nucleus of the cell. Cells break down the mRNA quickly after the mRNA is translated into the protein [32]. Due to this fact, mRNA vaccines are unlikely to pose a specific risk in pregnancy. The actual risks of mRNA vaccines to the pregnant person and her 
fetus are unknown because these vaccines have not been adequately studied in this population. In a cohort study evaluating the immunogenicity and reactogenicity of COVID-19 mRNA vaccination (BNT162b2 and mRNA-1273) in pregnant and lactating women, the vaccines induced antibody titers equivalent in pregnant and lactating women to nonpregnant women. These mRNA COVID-19 vaccines generated robust humoral immunity in pregnant and lactating women, similar to that observed in non-pregnant women. Further, the vaccine-induced immune responses were significantly greater than the response to natural infection, and the immune transfer to neonates was also observed via placenta and breast milk [33].

COVID-19 vaccine (Ad26.COV2.S) functions differently - in that the vaccine is a viral vector vaccine. This uses a viral vector to deliver the COVID-19 spike protein from which immunity is developed. Viral vectors have been previously given to pregnant patients of all trimesters [34] in large-scale Ebola vaccination trial and no adverse pregnancyrelated outcomes that affect the infant, were associated with viral vector vaccination in these trials. Currently, an openlabel, phase 2 study to evaluate the safety, reactogenicity, and immunogenicity of Ad26.COV2.S in healthy pregnant is ongoing. The purpose of this ongoing clinical trial is to assess the safety and reactogenicity of Ad26.COV2.S administered intramuscularly (IM) as a 2-dose schedule, in adult participants during the second and/or third trimester of pregnancy and (potentially) post-partum. This study is also going to assess the humoral immune response in peripheral blood of adult participants, to Ad26.COV2.S administered IM as a 2dose schedule during the second and/or third trimester of pregnancy, 28 days after the first and second vaccination.

In animals, development and reproductive toxicity (DART) studies using the mRNA (BNT162b2 and mRNA1273) and Ad26.COV2.S vaccines before or during pregnancy found no safety concerns. There were no direct or indirect harmful effects with respect to female reproduction, fetal/ embryonal development, or postnatal development [34].

The American College of Obstetricians and Gynecologists (ACOG) recommends COVID-19 vaccines should not be withheld from pregnant individuals who meet the criteria for vaccination based on the Advisory Committee on Immunization Practices (ACIP)-recommended priority groups.

\section{Limitations}

This review has potential limitations. Among them are a limited amount of large-scale studies due to the recency of the COVID-19 pandemic. Data collected from these studies also only reports of pregnant women in their third trimester. There is also a lack of published information on long-term outcomes in the post-partum period. Regardless of these limitations, this study has its strengths in compiling the current information on COVID-19 pregnancy, its risks, symptoms, diagnosis, and treatment.

\section{Conclusion}

The outbreak of COVID-19 has provided a challenge to all in the healthcare field. The lack of sufficient data and literature regarding the coronavirus has created an increased burden on providers and patients alike. This is especially evident in the pregnant population. There is limited evidence to provide clear-cut answers regarding recommendations for those at risk and infected with COVID-19 in the peripartum and antepartum period. Additionally, the evidence for this novel infection is conflicting and has not been in place long enough to truly assess the full length of pregnancy. Physicians must use their clinical judgment when evaluating the need for hospitalization and treatment in their pregnant patients since many symptoms of COVID-19 mimic those associated with normal physiologic changes in pregnancy. They must also take caution in counseling their patients regarding the outcomes both the patients and their developing fetuses may have after being affected. It will take years of data gathering to adequately state the risks to the mother and the fetus accurately, but prudency is recommended for all patients. Safe followup with physicians, early hospitalization when recommended, and protection of pregnant mothers during their third trimester are all ways to decrease the COVID-19 burden effectively.

Author Contribution V.H.: drafting of the review article, editing, interpretation of data, and revision. D.A.: drafting of the review article, editing, interpretation of data, and revision. D.K.: editing, interpretation of data, and review. P.K.: editing, interpretation of data, and review. K.J.: editing, interpretation of data, and review. M.P.: conceptualization, review, editing, and supervision of review article writing process.

Funding None.

Availability of Data and Material No data is available as it is a review article.

Code Availability N/A

\section{Declarations}

Ethics Approval N/A as it is a review article.

Consent to Participate N/A as it is a review article.

Consent for Publication N/A as it is a review article.

Conflict of Interest The authors declare no conflict of interest. 


\section{References}

1. CDC COVID Data Tracker. (n.d.). Available at: https://covid.cdc. gov/covid-data-tracker/ . Retrieved April $12^{\text {th }}, 2021$

2. Wong SF, Chow KM, Leung TN, Ng WF, Ng TK, Shek CC, et al. Pregnancy and perinatal outcomes of women with severe acute respiratory syndrome. Am J Obstet Gynecol. 2004;191(1):292-7.

3. Metcalfe J, Ueland K. Maternal cardiovascular adjustments to pregnancy. Prog Cardiovasc Dis. 1974;16(4):363-74.

4. Simon PM, Schwartzstein RM, Weiss JW, Fencl V, Teghtsoonian $\mathrm{M}$, Weinberger SE. Distinguishable types of dyspnea in patients with shortness of Breath1-3. Am Rev Respir Dis. 1990;142(5): 1009-14.

5. Huntley BJ, Huntley ES, Di Mascio D, Chen T, Berghella V, Chauhan SP. Rates of maternal and perinatal mortality and vertical transmission in pregnancies complicated by severe acute respiratory syndrome coronavirus 2 (SARS-Co-V-2) infection: a systematic review. Obstet Gynecol. 2020;136(2):303-12.

6. Sethuraman N, Jeremiah SS, Ryo A. Interpreting diagnostic tests for SARS-CoV-2. Jama. 2020;323(22):2249-51.

7. Wong HY, Lam HY, Fong AH, Leung ST, Chin TW, Lo CS, et al. Frequency and distribution of chest radiographic findings in patients positive for COVID-19. Radiology. 2020;296(2):E72-8.

8. Elshafeey F, Magdi R, Hindi N, Elshebiny M, Farrag N, Mahdy S, et al. A systematic scoping review of COVID-19 during pregnancy and childbirth. Int J Gynecol Obstet. 2020;150(1):47-52.

9. Dashraath P, Wong JL, Lim MX, Lim LM, Li S, Biswas A, et al. Coronavirus disease 2019 (COVID-19) pandemic and pregnancy. Am J Obstet Gynecol. 2020;222(6):521-31.

10. Khoury R, Bernstein PS, Debolt C, Stone J, Sutton DM, Simpson LL, et al. Characteristics and outcomes of 241 births to women with severe acute respiratory syndrome coronavirus 2 (SARS-CoV-2) infection at five New York City medical centers. Obstet Gynecol. 2020;136(2):273-82.

11. Di Mascio D, Khalil A, Saccone G, Rizzo G, Buca D, Liberati M, et al. Outcome of coronavirus spectrum infections (SARS, MERS, COVID-19) during pregnancy: a systematic review and meta-analysis. Am J Obstet Gynecol MFM. 2020;2(2):100107.

12. Kovac M, Mikovic Z, Rakicevic L, Srzentic S, Mandic V, Djordjevic V, et al. The use of D-dimer with new cutoff can be useful in diagnosis of venous thromboembolism in pregnancy. Eur J Obstet Gynecol Reprod Biol. 2010;148(1):27-30.

13. Ogawa $\mathrm{H}$, Asakura $\mathrm{H}$. Consideration of tranexamic acid administration to COVID-19 patients. Physiol Rev. 2020;100(4):1595-6.

14. Breslin N, Baptiste C, Gyamfi-Bannerman C, Miller R, Martinez R, Bernstein K, et al. COVID-19 infection among asymptomatic and symptomatic pregnant women: two weeks of confirmed presentations to an affiliated pair of New York City hospitals. Am J Obstet Gynecol MFM. 2(2):100118.

15. Kotlyar A, Grechukhina O, Chen A, Popkhadze S, Grimshaw A, Tal O, et al. Vertical transmission of COVID-19: a systematic review and meta-analysis. Am J Obstet Gynecol. 2020;31.

16. Zeng L, Xia S, Yuan W, Yan K, Xiao F, Shao J, et al. Neonatal early-onset infection with SARS-CoV-2 in 33 neonates born to mothers with COVID-19 in Wuhan, China. JAMA Pediatr. 2020;174(7):722-5.

17. Salvatore CM, Han JY, Acker KP, Tiwari P, Jin J, Brandler M, et al. Neonatal management and outcomes during the COVID-19 pandemic: an observation cohort study. Lancet Child Adolesc Health. 2020;4(10):721-7.
18. Henderson CE, Rezai S, Jackman JM. Universal screening for novel coronavirus disease 2019 (COVID-19) for asymptomatic parturients: may not be beneficial at this time. J Med Virol. 2021;93(3): 1198-200.

19. Hunt B, Retter A, McClintock C. Practical guidance for the prevention of thrombosis and management of coagulopathy and disseminated intravascular coagulation of patients infected with COVID19. Thrombosis UK: Llanwrda; 2020.

20. Wu Y, Zhang C, Liu H, Duan C, Li C, Fan J, et al. Perinatal depressive and anxiety symptoms of pregnant women during the coronavirus disease 2019 outbreak in China. Am J Obstet Gynecol. 2020;223(2):240-e1.

21. Narendra DK, Muigai D, Guntupalli KK. Acute respiratory distress syndrome in pregnancy. Critical Care Obstetrics. 2018;19:403.

22. Khan IH, Savarimuthu S, Leung MS, Harky A. The need to manage the risk of thromboembolism in COVID-19 patients. J Vasc Surg. 2020;14.

23. Royal College of Obstetricians \& Gynaecologists. Coronavirus (COVID-19) infection in pregnancy. Information for healthcare professionals. Version. 2020 Mar;4.

24. Yin S, Huang M, Li D, Tang N. Difference of coagulation features between severe pneumonia induced by SARS-CoV2 and nonSARS-CoV2. J Thromb Thrombolysis. 2020;3:1-4.

25. Hahambis TA, Burwick RM, Yawetz S, Stephenson KE, Collier AR, Sen P, Blackburn B, Kojic EM, Hirshberg A, Sobieszczyk ME, Marks K. Compassionate use of remdesivir in pregnant women with severe COVID-19. In2020 ACCP VIRTUAL Annual Meeting (October 19-30) 2020 Oct 27. American College of Clinical Pharmacy.

26. Group TR. Dexamethasone in hospitalized patients with Covid19-preliminary report. N Engl J Med. 2020;17.

27. Louchet M, Sibiude J, Peytavin G, Picone O, Tréluyer JM, Mandelbrot L. Placental transfer and safety in pregnancy of medications under investigation to treat COVID-19. Am J Obstet Gynecol MFM. 2020;22:100159.

28. Saad AF, Chappell L, Saade GR, Pacheco LD. Corticosteroids in the management of pregnant patients with coronavirus disease (COVID-19). Obstet Gynecol. 2020;136(4):823-6.

29. Brownfoot FC, Gagliardi DI, Bain E, Middleton P, Crowther CA Different corticosteroids and regimens for accelerating fetal lung maturation for women at risk of preterm birth. Cochrane Database Syst Rev. 2013;8.

30. Murphy VE, Fittock RJ, Zarzycki PK, Delahunty MM, Smith R, Clifton VL. Metabolism of synthetic steroids by the human placenta. Placenta. 2007;28(1):39-46.

31. Cheng X, Wang G. Ka Ho Lee K, Yang X. Dexamethasone use during pregnancy: potential adverse effects on embryonic skeletogenesis. Curr Pharm Des. 2014;20(34):5430-7.

32. Centers for Disease Control and Prevention. Understanding mRNA COVID-19 vaccines. Available at: https://www.cdc.gov/ coronavirus/2019-ncov/vaccines/different-vaccines/mrna.html.

33. Gray KJ, Bordt EA, Atyeo C, Deriso E, Akinwunmi B, Young N, et al. COVID-19 vaccine response in pregnant and lactating women: a cohort study. Am J Obstet Gynecol. 2021;26.

34. Vaccinating pregnant and lactating patients against COVID-19. Available at: https://www.acog.org/clinical/clinical-guidance/ practice-advisory/articles/2020/12/vaccinating-pregnant-andlactating-patients-against-covid-19

Publisher's Note Springer Nature remains neutral with regard to jurisdictional claims in published maps and institutional affiliations. 